\title{
Rician Noise Removal in Diffusion Tensor MRI
}

\author{
Saurav Basu, Thomas Fletcher, and Ross Whitaker \\ University of Utah, \\ School of Computing, \\ Salt Lake City, UT 84112, USA
}

\begin{abstract}
Rician noise introduces a bias into MRI measurements that can have a significant impact on the shapes and orientations of tensors in diffusion tensor magnetic resonance images. This is less of a problem in structural MRI, because this bias is signal dependent and it does not seriously impair tissue identification or clinical diagnoses. However, diffusion imaging is used extensively for quantitative evaluations, and the tensors used in those evaluations are biased in ways that depend on orientation and signal levels. This paper presents a strategy for filtering diffusion tensor magnetic resonance images that addresses these issues. The method is a maximum a posteriori estimation technique that operates directly on the diffusion weighted images and accounts for the biases introduced by Rician noise. We account for Rician noise through a data likelihood term that is combined with a spatial smoothing prior. The method compares favorably with several other approaches from the literature, including methods that filter diffusion weighted imagery and those that operate directly on the diffusion tensors.
\end{abstract}

\section{Introduction}

The quality of DT-MRI images is limited by the relatively long acquisition times necessary to obtain data at high spatial resolutions. Because acquisition time is restricted by issues of patient comfort and system demand, the signal-to-noise ratio(SNR) in DT-MRI is often low. Thus, post processing techniques to remove noise in the acquired data are important. The diffusion weighted images (DWIs), from which the tensors are derived, are corrupted by Rician noise, which introduces a positive bias in those measurements. These signal-dependent biases are not so detrimental to structural imaging, because they typically do not interfere with diagnostic decisions or tissue classification. However, DT-MRI measurements are being used extensively for quantitative comparisons, and several studies [123] have shown that bias can affect tensor properties such as trace and fractional anisotropy (FA).

Previous DT-MRI filtering methods fall into two categories: filters that operate on the DWIs and those that operate directly on the tensors. For instance Parker et al. [4] use Perona \& Malik (P\&M) 5] anisotropic diffusion to filter DWIs, and show that it produces less distortion in FA than filtering images of FA. Wang et al. 6] formulate a variational approach to regularize DWIs while 
constraining the estimated tensors to remain positive definite. Martin et al. [7] develop a Gaussian Markov Random Field model to regularize the estimated diffusion tensor images. Pennec et al. 8] describe a framework for performing anisotropic diffusion on tensors which preserves the property of tensors being symmetric and positive definite. Their filter is based on the idea that the space of all positive definite symmetric matrices forms a Riemannian manifold with each point representing a diffusion tensor. None of these techniques explicitly account for the effects of bias in the original DWI measurements. After submission of this work, we became aware of the work by Fillard et al. [9] that adds a Rician noise model to smoothing of tensor images in a Log-Euclidean framework.

In this paper we show Monte Carlo simulations that add new insights into the effects of Rician bias on tensor measurements. These results demonstrate the need for realistic noise models in DT-MRI filtering. We describe a filtering strategy that explicitly models the Rician noise as a data likelihood term in a maximum a posteriori framework. To assess the performance of our technique, we propose a new method for producing low noise DWIs using a maximum likelihood estimate (MLE) from repeated scans of a healthy volunteer. We present a comparison of filtering performance for tensor based methods and methods that smooth the DWIs. Our results show that filtering on the original DW images gives better results than filtering on tensor images, and that our method using an explicit model of Rician noise gives the best overall results.

\section{Rician Noise and Its Effects on Diffusion Tensors}

It is well known that MR magnitude images are corrupted by Rician noise, which arises from complex Gaussian noise in the original frequency domain ( $k$-space) measurements. The Rician probability density function for the corrupted image intensity $x$ is given by

$$
p(x)=\frac{x}{\sigma^{2}} \exp \left(-\frac{x^{2}+A^{2}}{2 \sigma^{2}}\right) I_{0}\left(\frac{x A}{\sigma^{2}}\right),
$$

where $A$ is the underlying true intensity, $\sigma$ is the standard deviation of the noise, and $I_{0}$ is the modified zeroth-order Bessel function of the first kind.

Previous studies on the effect of noise on diffusion tensor measurements have shown that as noise increases, the tensor trace decreases [1 and FA increases $\left.\begin{array}{llll}\hline 1 & 2 & 3\end{array}\right]$. Here we show that these effects can actually be quite different depending on the orientation of the diffusion tensor with respect to the measurement gradients. Using power series analysis, Anderson [2] shows that the major eigenvalue increases with higher noise, causing FA to increase. This analysis assumes the major eigenvalue is a combination of several diffusion weighted measurements, which happens when the major eigenvector lies in between several gradient directions. However, consider the special case of six gradient directions where the 

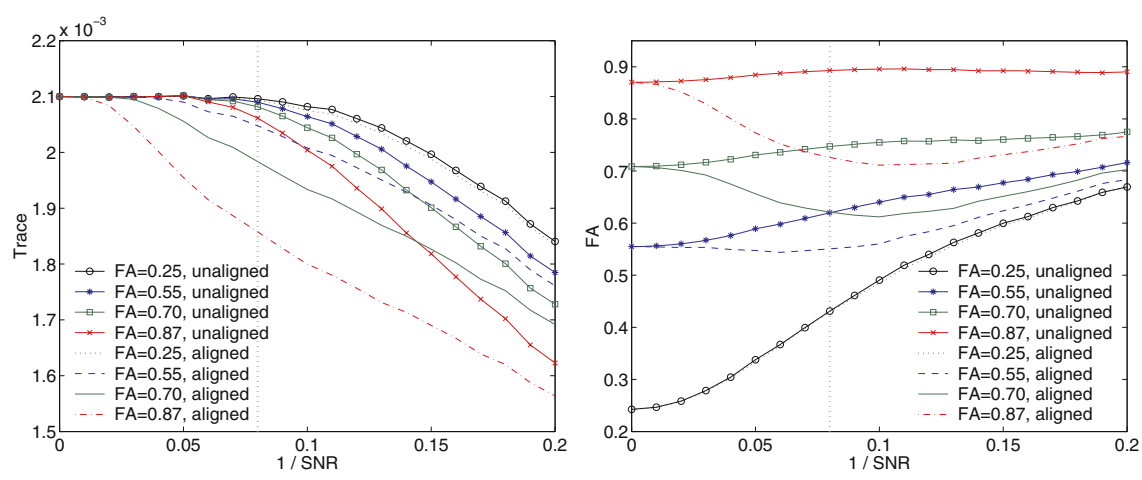

Fig. 1. Results of Monte Carlo simulations for trace (left) and FA (right). As an example, the SNR from the real data in section 4.2 is plotted as a vertical line.

major eigenvector is aligned with one gradient direction. Here the major eigenvalue will be completely determined from the diffusion weighted measurement in that direction. In this case, the Stejskal-Tanner equation for the $i$ th diffusion weighted value reduces to $A_{i}=A_{0} \exp \left(-b \lambda_{1}\left\|\mathbf{g}_{i}\right\|\right)$, where $\lambda_{1}$ is the major eigenvalue, $A_{0}$ is the baseline $\mathrm{T} 2$ value, and $\mathbf{g}_{i}$ is the gradient direction with which the major axis is aligned. Since $\lambda_{1}$ is large, the value $A_{i}$ will be much lower than the baseline $A_{0}$, and will thus be more susceptible to Rician bias. This positive bias will tend to underestimate the diffusion in the major eigendirection. We can thus expect two things: the underestimation effect of the tensor trace will be greater and FA will actually go down.

To test the hypothesis that tensor orientation changes the effects of noise on diffusion tensors, we performed Monte Carlo simulations of diffusion tensors characteristic of those found in brain white matter. These tensors had constant trace of $2.1 \times 10^{-3} \mathrm{~mm}^{2} / \mathrm{s}$ and four levels of fractional anisotropy, with eigenvalues $\lambda_{1}>\lambda_{2} \approx \lambda_{3}$. We used a $b$-value of $2000 \mathrm{~s} \mathrm{~mm}^{-2}$ and a commonly used sampling scheme of six gradient directions 10. We repeated the tests for two tensor orientations: one with major axis $(1,0,0)$, equally splitting the surrounding gradient directions, and one with the major axis aligned with a gradient direction. We varied the $1 / \mathrm{SNR}$ from 0 to 0.2 and used 10,000 tensors per trial.

Figure 1 shows the average trace and FA of the simulated tensors as a function of the noise level. Beyond what has previously been reported, our results show that the amount of bias in the trace increases when the tensor has higher anisotropy or when the tensor is aligned with a gradient direction. Our results show that tensors that are not aligned with gradient directions tend to have increased FA, while those aligned with a gradient direction tend to have decreased FA for moderate levels of noise. However, for lower $b$-values (e.g., $b=1000)$ these effects are less pronounced. The fact that FA can be overestimated or underestimated depending on the orientation of the fiber tract in the scanner has serious consequences for clinical studies using DT-MRI. 


\section{Rician Bias Correction Filter}

Our Rician bias correction filter is based on a maximum a posteriori (MAP) approach to the image reconstruction problem. Given an initial noisy image $u_{0}$, we construct the filtered image $u$ that maximizes the log-posterior probability

$$
\log p\left(u \mid u_{0}\right) \propto \log p\left(u_{0} \mid u\right)+\log p(u),
$$

where $p\left(u_{0} \mid u\right)$ is the likelihood term, or noise model, and $p(u)$ is the prior. For DWIs we consider $u$ to be a vector-valued image, each gradient direction (including $b=0$ ) representing a vector component. The formulation in this section would also be valid for structural MRI.

\subsection{The Rician Likelihood Term}

The formulation of the filtering problem as maximization of a posterior $p\left(u \mid u_{0}\right)$ is useful as it allows us to incorporate the Rician bias correction as a data attachment term which can be added to the prior model. Using the Rician distribution (11) as the likelihood term and assuming independent noise, the pointwise loglikelihood becomes

$$
\log p\left(u_{0} \mid u\right)=\log \frac{u_{0}}{\sigma^{2}}-\frac{u_{0}^{2}+u^{2}}{2 \sigma^{2}}+\log I_{0}\left(\frac{u_{0} u}{\sigma^{2}}\right) .
$$

The derivative of (3) with respect to $u$, gives Rician data attachment term

$$
\mathcal{B}=-\frac{u}{\sigma^{2}}+\left[I_{1}\left(\frac{u_{0} u}{\sigma^{2}}\right) / I_{0}\left(\frac{u_{0} u}{\sigma^{2}}\right)\right] \frac{u_{0}}{\sigma^{2}} .
$$

\subsection{Combining the Rician Model with a Prior}

The data likelihood term can be combined with any image prior model. In this paper we use a Gibb's prior model based on a P\&M energy functional, given by

$$
p(u)=\frac{1}{z} \exp (-E(u)), \quad E(u)=\lambda \int_{U} c\left(\|\nabla u\|^{2}\right) d x d y,
$$

where $z$ is a suitable normalization, $U$ is the image domain and c is the conductance given by $c\left(\|\nabla u\|^{2}\right)=\exp \left(-\|\nabla u\|^{2} / 2 k^{2}\right), \mathrm{k}$ is the conductance parameter and $\lambda$ is a constant weighting term.

By adding the Rician likelihood term (44) with the variational of the P\&M energy functional we form the update equation for the filtered image,

$$
\frac{\partial u}{\partial t}=\mathcal{B}+\lambda \operatorname{div}\left(c\left(\|\nabla u\|^{2}\right) \nabla u\right) .
$$




\section{Results}

The performance comparisons were made on four different filtering methods: Euclidean tensor filtering, Riemannian tensor filtering [8] and vector anisotropic diffusion on DWIs with and without the Rician likelihood term as described in section 3.1. Here Euclidean filtering refers to vector anisotropic diffusion on the tensor components. To compare these methods, we used both synthetic and real datasets. We used three different error metrics - root mean squared (RMS) error in the tensor, trace and fractional anisotropy. The error between tensors is computed using the Frobenius norm. The parameters for each method were optimized for the RMS error on the tensor components. Both synthetic and real datasets use seven images for each slice, one without diffusion gradient $(b=0)$ and the remaining six with $b=1000 \mathrm{~s} / \mathrm{mm}^{2}$ and diffusion gradients along the standard six orientations [10].

\subsection{Synthetic Data}

We used a $10 \times 10 \times 4$ volume of tensors oriented in two directions so as to have a tensor discontinuity. One group of tensors have major axes that split the gradient directions, while the others are aligned with a gradient. Figure 2 shows the clean and noisy synthetic tensors with $\mathrm{SNR}=15$.

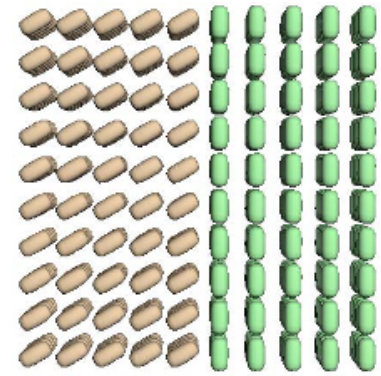

Clean Data

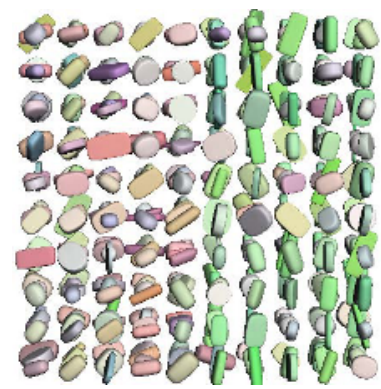

Noisy Data $(\mathrm{SNR}=15)$

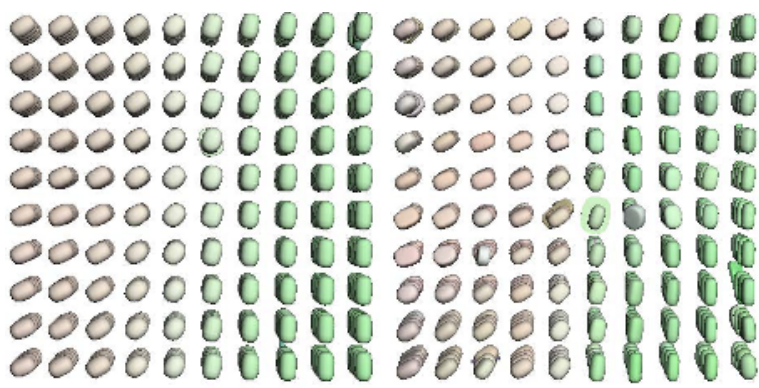

Euclidean

Riemannian

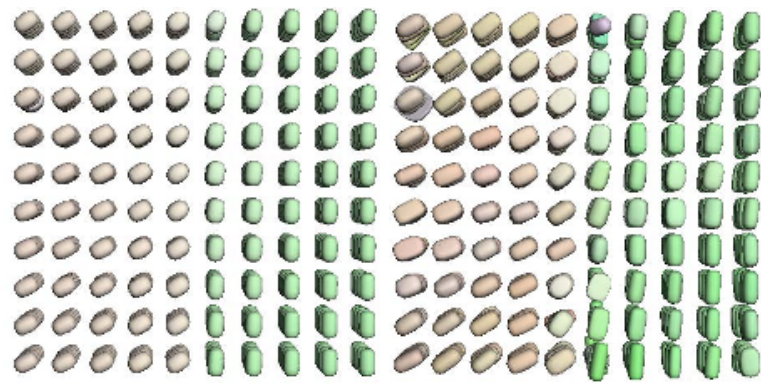

Vector Aniso-DWI

Rician -DWI

Fig. 2. Synthetic Data Filter Results 

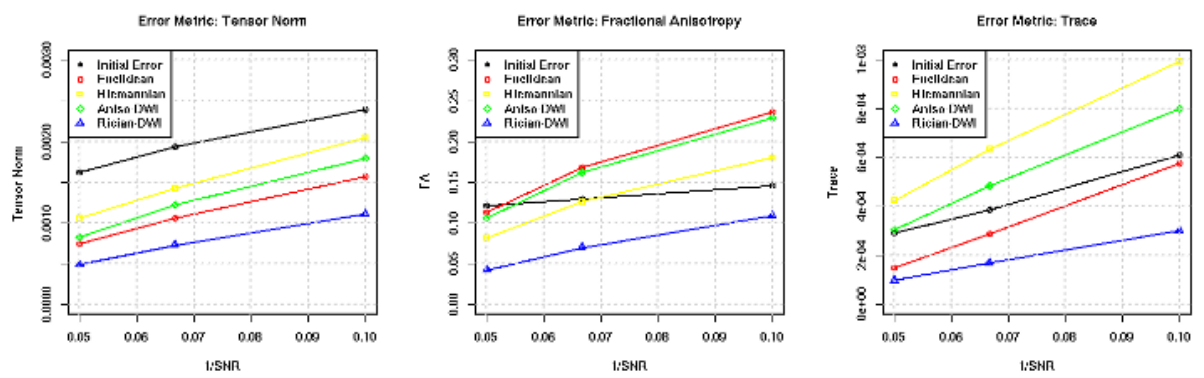

Fig. 3. Plots of error metrics for the various filters on synthetic data
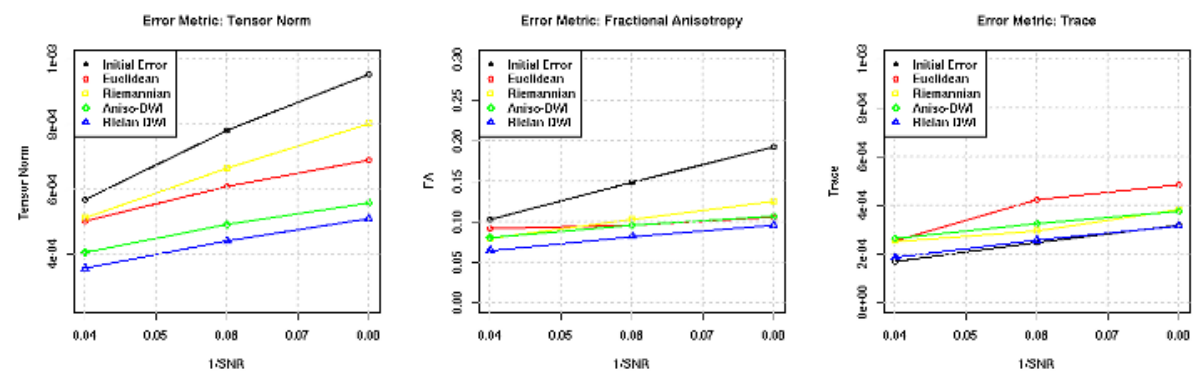

Fig. 4. Plots of error metrics for the various filters on real data

\subsection{Real Data}

DTI Ground Truth Generation: A key challenge in quantitatively evaluating filtering methods on real diffusion tensor data is the lack of a ground truth. While realistic simulated brain datasets exists for structural MRI, no such normalized data is available for DT-MRI. For this paper we develop a new approach for generating high SNR diffusion weighted image data. This technique builds a ground truth image as a maximum likelihood (ML) estimate from a set of repeated scans of the same subject. If $\left\{x_{i}\right\}$ is the set of intensities from the same voxel in $N$ repeated scans, then the ML estimate of the true intensity $A$ is found by maximizing the $\log$-likelihood, $\log L=\sum_{i=1}^{N} \log p\left(x_{i} \mid A\right)$, where $p\left(x_{i} \mid A\right)$ is the Rician pdf as given in (11). The properties of the ML estimate are investigated by Sijbers et al. [11]. This ML estimate is superior to a simple averaging of the intensities as it incorporates a priori knowledge of the noise statistics. Also, it is well known that in the limit the ML estimate is most precise.

About the data: We generated our ground truth ML images from a set of five scans of a healthy volunteer on a Siemens head-only 3T scanner (Allegra). For each sequence, a single shot echo planar (EPI) diffusion tensor sequence with total scan time of approximately 12 minutes was used. The imaging parameters were: $\mathrm{TR}=5400 \mathrm{~ms}, \mathrm{TR}=73 \mathrm{~ms}$, isotropic voxels with $2 \mathrm{~mm}$ slice distance and inplane resolution $=2 \times 2 \mathrm{~mm}, 20$ averages. We added known Rician noise to the 

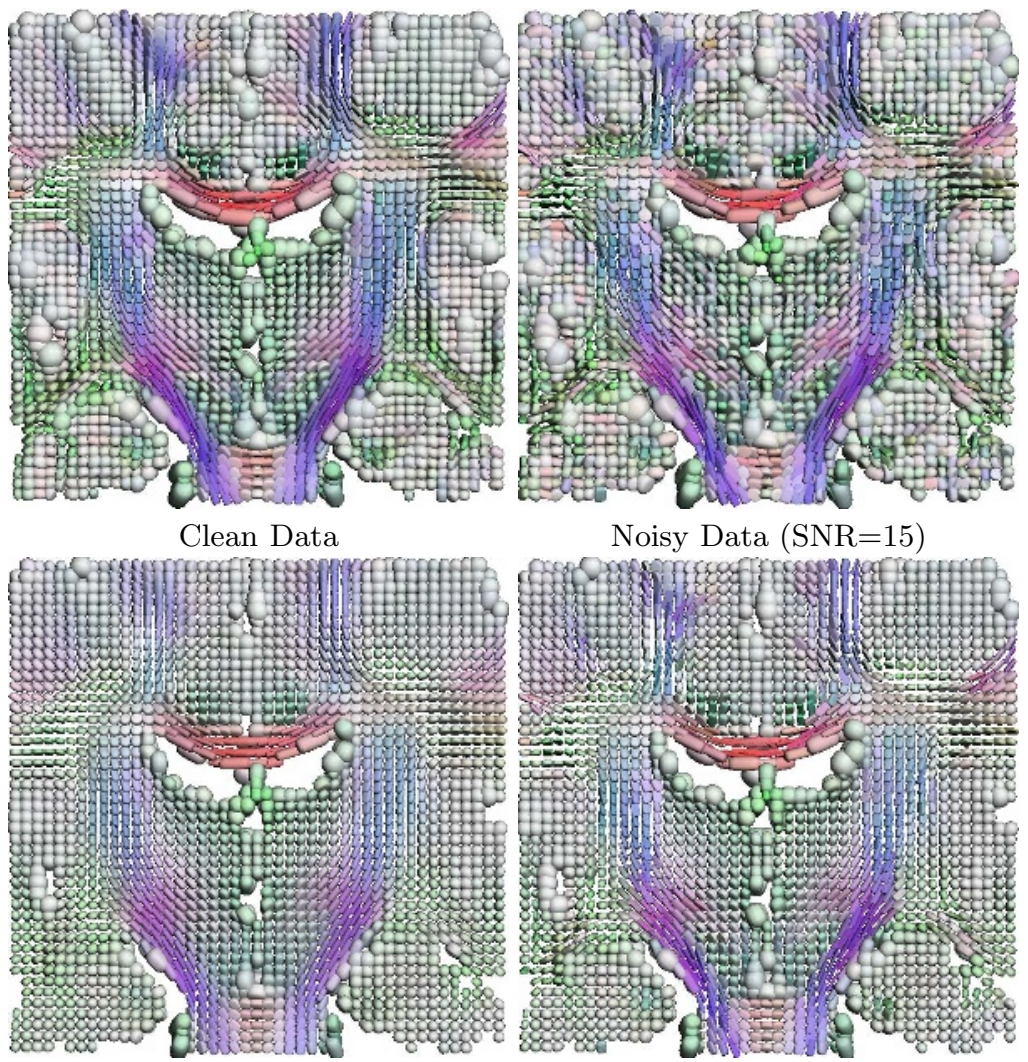

Euclidean

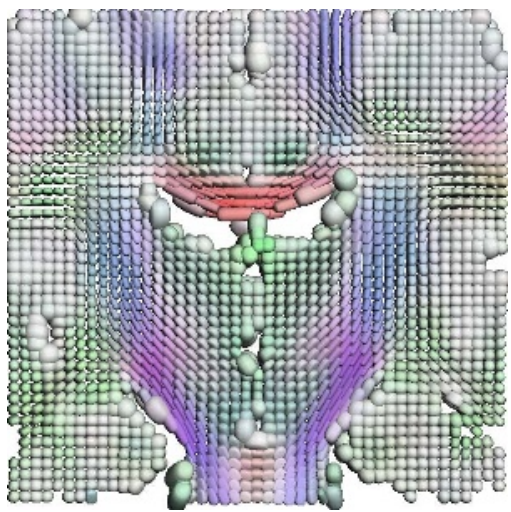

Vector Aniso-DWI

Riemannian

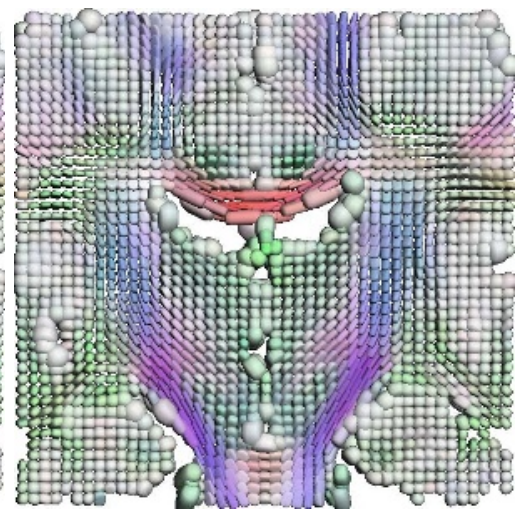

Rician -DWI

Fig. 5. Real Data Filtering Results

ML estimated DWIs at SNR levels of 10, 15 and 20 with respect to the average white matter signal level in the $b=0$ image. A slice from the ground truth and $\mathrm{SNR}=15$ tensor images is shown in Figure 5 . 


\subsection{Performance}

The resulting error metrics for the various filtering methods on the synthetic and real data are shown in Figures 3 and 4 . The original, noisy and filtered images for $\mathrm{SNR}=15$ are shown with superquadric glyphs 12 in Figures 2 and 5. The results demonstrate that the Rician filter with the bias correction term gives better RMS error on tensor components. On both the real and synthetic data the Rician filter is superior to all the other filtering techniques.The data also shows that for most of the error metrics the filtering methods on the DWI's yields better results than smoothing on the tensor space. The Riemannian filter requires all tensors to be positive definite and is thus disadvantaged by the process of adjusting for negative eigenvalues.

\section{Conclusions}

We presented a new method for denoising diffusion tensor images that includes a Rician noise model as part of MAP estimation framework. To the best of our knowledge, this is the first work to explicitly model and remove the bias effects of Rician noise in DT-MRI. We presented Monte Carlo simulations that show that noise can distort tensors in a manner that is dependent on the orientation and anisotropy of the underlying tensor. Our filtering results demonstrated that filtering on the original DWIs yields superior results to filtering methods that operate on the estimated tensors. Filtering on the DWIs with our Rician noise model gave the best overall results.

\section{Acknowldgments}

This work is part of the National Alliance for Medical Image Computing (NAMIC), funded by the National Institutes of Health through the NIH Roadmap for Medical Research, Grant U54 EB005149. Information on the National Centers for Biomedical Computing can be obtained from http://nihroadmap.nih.gov/ bioinformatics. Funding for this work has also been provided by Center for Integrative Biomedical Computing, NIH NCRR Project 2-P41-RR12553-07. We thank Weili Lin and Guido Gerig from the University of North Carolina for providing us with the DW-MRI data. Glyph visualizations created with Teem (http://teem.sf.net).

\section{References}

1. Pierpaoli, C., Basser, P.: Toward a quantitative assessment of diffusion anisotropy. Magnetic Resonance in Medicine 36(6) (1996) 893-906

2. Anderson, A.W.: Theoretical analysis of the effects of noise on diffusion tensor imaging. Magnetic Resonance in Medicine 46(6) (2001) 1174-1188

3. Skare, S., Li, T., Nordell, B., Ingvar, M.: Noise considerations in the determination of diffusion tensor anisotropy. Magnetic Resonance Imaging 18(6) (2000) 659-669 
4. J., P.G.: Nonlinear smoothing for reduction of systematic and random errors in diffusion tensor imaging. J Magn. Reson Imaging 11(6) (2000) 702-710

5. Perona, P., Malik, J.: Scale-space and edge detection using anisotropic diffusion. IEEE Transactions on Pattern Analysis Machine Intelligence 17(4) (1990) 629-639

6. Wang, Z., Vemuri, B., Chen, Y., Mareci, T.: A constrained variational principle for direct estimation and smoothing of the diffusion tensor field from complex DWI. IEEE Transactions on Medical Imaging 23(8) (2004) 930-939

7. Martin Fernandez, C.F. Westin, C.A.L.: 3d bayesian regularization of diffusion tensor mri using multivariate gaussian markov random fields. In: Medical Image Computing and Computer-Assisted Intervention (MICCAI'04). (2004)

8. Pennec, X., Fillard, P., Ayache, N.: A riemannian framework for tensor computing. International Journal of Computer Vision 66(1) (2006) 41-66

9. Fillard, P., Arsigny, V., Pennec, X., Ayache, N.: Clinical DT-MRI estimation, smoothing and fiber tracking with log-Euclidean metrics. In: Proceedings of the Third IEEE International Symposium on Biomedical Imaging (ISBI 2006), Crystal Gateway Marriott, Arlington, Virginia, USA (2006) 786-789

10. Basser, P., Pierpaoli, C.: Microstructural and physiological features of tissues elucidated by quantitative-diffusion-tensor MRI. J. Mag Res. 111(3) (1996) 209-219

11. Sijbers, J., den Dekker, A., Scheunders, P., Dyck, D.V.: Maximum-Likelihood Estimation of Rician Distribution Parameters. IEEE Transactions on Medical Imaging 17(3) (1998) 357-361

12. Kindlmann, G.: Superquadric tensor glyphs. In: Proceedings of IEEE TVCG/EG Symposium on Visualization 2004. (2004) 147-154 